\title{
Circular RNA expression profile in human primary multiple intracranial aneurysm
}

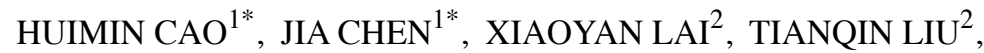 \\ PING QIU $^{2}$, SHUANGLIN QUE ${ }^{2}$ and YANMING HUANG ${ }^{2}$ \\ ${ }^{1}$ Central Laboratory, ${ }^{2}$ Department of Neurosurgery, Longyan First Hospital \\ Affiliated to Fujian Medical University, Longyan, Fujian 364000, P.R. China
}

Received April 14, 2020; Accepted December 8, 2020

DOI: $10.3892 / \mathrm{etm} .2021 .9670$

\begin{abstract}
Primary multiple intracranial aneurysm (MIA) is a vascular disease that frequently leads to fatal vascular rupture and subarachnoid hemorrhage. However, the epigenetic regulation associated with MIA has remained largely elusive. Circular RNAs (circRNAs) serve important roles in cardiovascular diseases; however, their association with MIA has remained to be investigated. The present study initially aimed to explore novel mechanisms of MIA through examining circRNA expression profiles. Comprehensive circRNA expression profiles were detected by RNA sequencing (RNA-Seq) in human peripheral blood mononuclear cells. The RNA-Seq results were validated by reverse transcription-quantitative PCR. Gene Ontology and Kyoto Encyclopedia of Genes and Genomes (KEGG) analyses suggested the functions of these circRNAs. A competing endogenous RNA network was constructed to reveal the circRNA-microRNA-mRNA relationship. Among the 3,328 differentially expressed circRNAs between the MIA and matched control groups, 60 exhibited significant expression changes ( $\mid \log 2$ fold changel $\geq 2$; $\mathrm{P}<0.05)$. Among these 60 circRNAs, 20 were upregulated,
\end{abstract}

Correspondence to: Dr Yanming Huang, Department of Neurosurgery, Longyan First Hospital Affiliated to Fujian Medical University, 105 Jiuyi North Road, Xinluo, Longyan, Fujian 364000, P.R. China

E-mail: liochristine@icloud.com

${ }^{*}$ Contributed equally

Abbreviations: MIA, multiple intracranial aneurysm; circRNA, circular RNA; miRNA, microRNA; lncRNA, long non-coding RNA; GO, Gene Ontology; KEGG, Kyoto Encyclopedia of Genes and Genomes; ceRNA, competing endogenous RNA; CTA, computed tomography angiography; DSA, digital subtraction angiography; LFA-1, lymphocyte function-associated antigen-1; ICAM-1, intercellular cell adhesion molecule-1

Key words: primary multiple intracranial aneurysm, circular RNA, gene expression profiling, inflammation, leukocyte transendothelial migration while the other 40 were downregulated. A number of downregulated circRNAs were involved in inflammation. The most significant KEGG pathway was 'leukocyte transendothelial migration'. The circRNAs Homo sapiens (hsa)_circ_0135895, hsa_circ_0000682 and hsa_circ_0000690, which were also associated with the above-mentioned pathway, were indicated to be able to regulate protein tyrosine kinase 2 , protein kinase $C \beta$ and integrin subunit $\alpha \mathrm{L}$, respectively. To the best of our knowledge, the present study was the first to perform a circRNA sequencing analysis of MIA. The results specifically predicted the regulatory role of circRNAs in the pathogenesis of MIA. 'Leukocyte transendothelial migration' may be critical for the pathogenesis of MIA.

\section{Introduction}

Primary intracranial aneurysm (IA) is a vascular disease that frequently leads to fatal vascular rupture and subarachnoid hemorrhage, which is an acute stroke and has a mortality rate of nearly $50 \%$ (1). Primary multiple IA (MIA) is defined as the presence of two or more aneurysms in one patient. The reported rates of MIA range between 2 and $45 \%$ of IAs (2).

With the accumulation of studies about immunization, non-coding RNAs and epigenetics, the molecular mechanisms of IA have been widely identified. However, although numerous studies have explored microRNAs (miRNAs) (3-5) or long non-coding RNAs (lncRNAs) $(6,7)$ in IA, circRNAs in IA, particularly in MIA, have remained largely elusive. The genetic pathology of MIA, particularly in terms of circular RNAs (circRNAs) in MIA, remains to be determined.

Since their 3 and 5' ends are connected to form a circle, circRNA molecules cannot be broken down by RNase R. This feature leads to their high stability and abundance. Furthermore, circRNA expression is frequently cell- or tissue-specific. In addition, circRNAs may be potential biomarkers for diseases (8), as they are conserved between species. Previous studies have demonstrated that circRNAs have numerous functions, such as sponging of miRNAs and gene regulation, and are closely associated with cell function and diseases. Due to rapid developments in sequencing and microarray technology, systematic investigations of circRNA expression profiles in MIA development are now possible. 
Using high-throughput sequencing technology, the present study aimed to define the circRNA expression profiles of MIA and to explore novel pathological mechanisms of MIA. Compared with microarrays, sequencing technology provides more comprehensive and reliable data (9), which may improve the current knowledge of the epigenetic mechanisms of MIA. The present study revealed that certain circRNAs are involved in the pathogenesis of MIA and mostly associated with inflammation. These results were further verified using PCR assays in an enlarged cohort of patients with MIA. In summary, the present results may give novel insight into the molecular mechanisms of MIA, provide ideas for novel treatments and promote the exploration of circRNAs as biomarkers.

\section{Materials and methods}

Patient selection. Blood samples of three pairs of patients with primary MIA and matched healthy individuals were collected at the Department of Neurosurgery, Longyan First Hospital Affiliated to Fujian Medical University (Longyan, China) between April 2018 and June 2018. Patients in the MIA group were selected from patients with onset within $24 \mathrm{~h}$ who were confirmed to have one ruptured IA by head computed tomography angiography (CTA) or digital subtraction angiography (DSA). The patients received microsurgical clipping or interventional therapy within the acute phase $(24 \mathrm{~h})$ after one of the aneurysms was ruptured. Those patients who had cerebrovascular diseases other than IA, systemic malignant tumor or severe complications were excluded. Individuals in the matched group were selected among individuals who were attending a regular health check-up and underwent head CTA/DSA to exclude IA. To ensure the quality of samples, the present study excluded patients with factors that may have affected the state of peripheral blood, including pregnancy, chemotherapy and fever $\left(\geq 37.3^{\circ} \mathrm{C}\right)$. Individuals in the MIA and matched groups were matched based on age, sex and past medical history, such as hypertension and smoking. Baseline information and clinical characteristics of the two groups are presented in Table I. Another 20 individuals were recruited as the validation cohort following the same criteria as those for the initial study group used for RNA sequencing (RNA-seq). They were divided into 10 pairs of matched patients with primary MIA as a test group and healthy individuals as a control group. The baseline information and clinical features of the two groups are presented in Table II. All volunteers included provided written informed consent and the present study was approved by the Ethics Committee of Longyan First Hospital Affiliated to Fujian Medical University (Longyan, China).

RNA isolation and purification. Total RNA was extracted from peripheral blood mononuclear cell samples using TRlzol reagent (Takara Bio, Inc.) according to the kit's instructions, as described previously (10). The NanoDrop-1000 (Thermo Fisher Scientific, Inc.) was used to quantify total RNA, and optical density at $260 \mathrm{~nm}$ (OD260)/OD280 ratios between 1.8 and 2.1 were considered acceptable. Agarose gel electrophoresis and the Nanodrop spectrophotometer were used to check the quantity of RNA prior to sequencing and reverse transcription-quantitative PCR (RT-qPCR).
circRNA sequencing analysis. RNase R (Epicentre; Illumina, Inc.) was used to degrade linear RNA and enrich circRNAs in total RNA. Subsequently, the circRNA library was constructed according to the manufacturer's protocols (NEBNext Ultra Directional RNA Library Prep kit; New England BioLabs, Inc.). The Library Quantification kit (Kapa Biosystems; Roche Diagnostics) was used to identify and quantify sequences using the Illumina HiSeq 4000 system (Illumina, Inc.). By using paired-end sequencing, the nucleotide length of sequencing was $150 \mathrm{bp}$. Single-stranded DNA was generated using $0.1 \mathrm{M}$ $\mathrm{NaOH}$ and its loading concentration was $8 \mathrm{pM}$, which was measured by qPCR $(11,12)$. Sequencing quality was evaluated by FastQC v0.11.7 software (http://www.bioinformatics. babraham.ac.uk/projects/fastqc/). Adaptors and poor-quality bases were trimmed with Cutadapt v1.14 (https://cutadapt. readthedocs.io/en/stable/). In order to ensure the quality and reliability of data analysis, it was necessary to filter the original data. The filtering comprised the following: Reads with adapter were removed; reads with a ratio of indeterminable base information of $>0.002$ were removed; when the number of low-quality bases in a single-ended read exceeded $50 \%$ of read length, this paired read was removed. Furthermore, genes with low expression levels were filtered in the analysis. The threshold values were as follows: 1.5 -fold change (FC), $\mathrm{P} \leq 0.05$ and mean value of fragments per kilobase of exon model per million reads mapped $\geq 0.5$.

Base calling and image processing were performed using Solexa pipeline v1.8 (Off-Line Base Caller software, v1.8). Quantile normalization and subsequent data processing were performed using the Ballgown package v2.8.4 (http://www. bioconductor.org/packages/release/bioc/html/ballgown.html) in R software v3.4.1 (www.r-project.org). Differential expression patterns of circRNAs in the samples were visualized using hierarchical clustering and a scatter plot. Significantly differentially expressed circRNAs ( $\mathrm{FC}>2$ and $\mathrm{P}<0.05$ ) were identified using volcano plot filtering.

Validation by $R T-q P C R$. To validate the changes in circRNA expression identified by RNA-sequencing, 10 circRNAs, including 5 upregulated and 5 downregulated circRNAs, were selected. They met the following requirements: i) Length of circRNA between 200 and $3,000 \mathrm{bp}$; ii) $\log 2 \mathrm{FCl}>2$; iii) $\mathrm{P}<0.05$; and iv) exon-related circRNA. The expression levels of circRNAs were evaluated by fluorescence real-time PCR (C1000; Applied Biosystems; Thermo Fisher Scientific, Inc.) in 10 pairs of matched patients with primary MIA as a test group and healthy individuals as a control group using SYBR Green as the probe, as previously described (13). The housekeeping gene $\beta$-actin was used as an internal control. The results were calculated using the $2^{-\Delta \Delta \mathrm{Cq}}$ method (14).

Gene Ontology (GO) and Kyoto Encyclopedia of Genes and Genomes (KEGG) analysis. GO enrichment analysis (https://david-d.ncifcrf.gov/) was conducted to explore the potential biological functions of the target genes of the circRNAs identified. KEGG pathway analysis (http://www. genome.jp/kegg/) revealed the signaling pathways the target genes of identified circRNAs were involved in at the molecular level. Genes with a corrected $\mathrm{P}<0.05$ were considered to be enriched. 
Table I. Baseline data of patients for sequencing and the clinical features of MIA.

A, Clinicopathological parameters

\begin{tabular}{|c|c|c|c|c|}
\hline Item & $\operatorname{MIA}(n=3)$ & Matched control $(n=3)$ & $\mathrm{t} / \chi^{2}$ & P-value \\
\hline Age (years) & $69.33 \pm 8.39$ & $68.67 \pm 9.29$ & 0.092 & 0.931 \\
\hline Female sex & $3(100)$ & $3(100)$ & 0.000 & 1.000 \\
\hline Hypertension & $3(100)$ & $3(100)$ & 0.000 & 1.000 \\
\hline Smoking & $0(100)$ & $0(100)$ & 0.000 & 1.000 \\
\hline $\mathrm{SBP}(\mathrm{mmHg})$ & $156.67 \pm 10.60$ & $153.00 \pm 11.79$ & 0.401 & 0.709 \\
\hline DBP (mmHg) & $83.67 \pm 5.13$ & $84.33 \pm 9.07$ & -0.111 & 0.917 \\
\hline TG (mmol/l) & $0.67 \pm 0.05$ & $0.75 \pm 0.07$ & -1.595 & 0.186 \\
\hline LDL (mmol/l) & $1.81 \pm 0.16$ & $1.88 \pm 0.13$ & -0.632 & 0.561 \\
\hline $\mathrm{HDL}(\mathrm{mmol} / \mathrm{l})$ & $1.33 \pm 0.10$ & $1.38 \pm 0.14$ & -0.511 & 0.636 \\
\hline
\end{tabular}

B, Details of individual IAs

\begin{tabular}{|c|c|c|c|c|}
\hline Case/IA & Location & Ruptured & Type & Size $(\mathrm{mm})$ \\
\hline \multicolumn{5}{|l|}{ Case 1} \\
\hline IA 1 & Right PcoA & Yes & Saccular & $3 \times 5$ \\
\hline IA2 & Left PcoA & No & Saccular & $2 \times 5$ \\
\hline \multicolumn{5}{|l|}{ Case 2} \\
\hline IA1 & BA & Yes & Saccular & $7 \times 9$ \\
\hline IA2 & Left MCA & No & Saccular & $3 \times 4$ \\
\hline \multicolumn{5}{|l|}{ Case 3} \\
\hline IA1 & Right PcoA & Yes & Saccular & $9 \times 3$ \\
\hline IA 2 & AcoA & No & Saccular & $3 \times 4$ \\
\hline
\end{tabular}

Values are expressed as $\mathrm{n}(\%)$ or the mean \pm standard deviation. MIA, multiple intracranial aneurysm; SBP, systolic blood pressure; DBP, diastolic blood pressure; TG, triglyceride; LDL, low-density lipoprotein; HDL, high-density lipoprotein; PcoA, posterior communicating artery aneurysm; BA, basal artery aneurysm; MCA, middle cerebral artery; AcoA, anterior communicating artery.

miRNA prediction and circRNA-miRNA-mRNA network construction. miRNA binding sites of circRNAs were predicted using TargetScan v7.2 (15), circBank v2014 (16) and miRanda v3.3a. Information on miRNA-mRNA regulation was obtained using miRTarBase (17) and TargetScan. The potential effects of the differentially expressed circRNAs associated with 'leukocyte transendothelial migration' were further predicted by constructing a competing endogenous RNA (ceRNA) network of circRNA-miRNA-mRNA interactions. Cytoscape v3.6.1 was used to construct the graph of the circRNA-miRNA-mRNA network.

Statistical analysis. Normally distributed data were analyzed using a two-tailed t-test and a Mann-Whitney U test was used for skewed data. All statistical analyses were performed using SPSS v19.0 (IBM Corp.). P<0.05 (two-tailed) was considered to indicate a statistically significant difference.

\section{Results}

circRNA expression profiles in peripheral blood mononuclear cells. The normalized expression profiles are presented in
Fig. 1. Hierarchical clustering revealed the difference in circRNA expression profiles between the two groups (Fig. 1A). A total of 3,328 differentially expressed circRNAs between the MIA group and the matched group were identified by circRNA sequencing analysis. The distribution of circRNAs in three pairs of matched samples is presented in a scatter plot (Fig. 1B). Compared to the matched group, 60 circRNAs were revealed to be significantly differentially expressed in the MIA group ( $\log 2 \mathrm{FCl} \geq 2 ; \mathrm{P}<0.05$ ). Among them, 20 were upregulated and 40 were downregulated (Fig. 1C). Furthermore, $85.79 \%$ of the circRNAs were previously identified in circbase (18) and $14.21 \%$ were novel (Fig. 1D). Most circRNAs had a predicted spliced length $<1,000$ nt (Fig. 1E). These circRNAs were widely distributed among all chromosomes except the $\mathrm{Y}$ chromosome, since only samples from female patients were collected (Fig. 1F). The top 10 most upregulated and downregulated circRNAs are listed in Table III.

$R T$-qPCR validation of differential expression of circRNAs. A total of 10 differentially expressed circRNAs, including 5 upregulated [Homo sapiens circRNA (hsa_circ)_0008911, hsa_circ_0074837,hsa_circ_0131628,hsa_circ_0083229 and 
Table II. Baseline data of the two groups of the validation cohort and the clinical features of multiple IA.

A, Baseline data of the test group and the control group of the validation cohort

\begin{tabular}{|c|c|c|c|c|}
\hline Item & Test group $(n=10)$ & Control $(n=10)$ & $t / \chi^{2}$ & P-value \\
\hline Age (years) & $57.0 \pm 9.31$ & $59.8 \pm 8.99$ & -0.684 & 0.503 \\
\hline Female sex & $6(60)$ & $6(60)$ & 0.000 & 1.000 \\
\hline Hypertension & $8(80)$ & $8(80)$ & 0.000 & 1.000 \\
\hline Smoking & $6(60)$ & $6(60)$ & 0.000 & 1.000 \\
\hline $\mathrm{SBP}(\mathrm{mmHg})$ & $147.60 \pm 14.42$ & $149.40 \pm 18.31$ & -0.244 & 0.810 \\
\hline DBP (mmHg) & $83.90 \pm 4.68$ & $84.20 \pm 8.94$ & -0.094 & 0.926 \\
\hline $\mathrm{TG}(\mathrm{mmol} / \mathrm{l})$ & $0.70 \pm 0.08$ & $0.71 \pm 0.09$ & -0.274 & 0.787 \\
\hline $\mathrm{LDL}(\mathrm{mmol} / \mathrm{l})$ & $1.78 \pm 0.15$ & $1.82 \pm 0.18$ & -0.518 & 0.610 \\
\hline $\mathrm{HDL}(\mathrm{mmol} / \mathrm{l})$ & $1.41 \pm 0.10$ & $1.35 \pm 0.08$ & 1.414 & 0.174 \\
\hline
\end{tabular}

B, Details of individual IAs

\begin{tabular}{|c|c|c|c|c|}
\hline Subject/IA & Location & Ruptured & Type & Size $(\mathrm{mm})$ \\
\hline \multicolumn{5}{|l|}{ Test 1} \\
\hline IA1 & Right PcoA & Yes & Saccular & $4 \times 4$ \\
\hline IA 2 & Left PcoA & No & Saccular & $4 \times 2$ \\
\hline \multicolumn{5}{|l|}{ Test 2} \\
\hline IA1 & BA & Yes & Saccular & $6 \times 5$ \\
\hline IA 2 & Left MCA & No & Saccular & $4 \times 3$ \\
\hline \multicolumn{5}{|l|}{ Test 3} \\
\hline IA1 & Right PcoA & Yes & Saccular & $5 \times 3$ \\
\hline IA 2 & $\mathrm{Aco} A$ & No & Saccular & $5 \times 2$ \\
\hline \multicolumn{5}{|l|}{ Test 4} \\
\hline IA1 & Left PcoA & Yes & Saccular & $8 \times 7$ \\
\hline IA2 & Right PcoA & No & Saccular & $6 \times 4$ \\
\hline \multicolumn{5}{|l|}{ Test 5} \\
\hline IA1 & Left PcoA & Yes & Saccular & $12 \times 6$ \\
\hline IA 2 & Left MCA & No & Saccular & $5 \times 2$ \\
\hline \multicolumn{5}{|l|}{ Test 6} \\
\hline IA1 & Left MCA & Yes & Saccular & $9 \times 7$ \\
\hline IA2 & AcoA & No & Saccular & $7 \times 6$ \\
\hline \multicolumn{5}{|l|}{ Test 7} \\
\hline IA1 & AcoA & Yes & Saccular & $13 \times 10$ \\
\hline IA 2 & Right PcoA & No & Saccular & $9 \times 6$ \\
\hline \multicolumn{5}{|l|}{ Test 8} \\
\hline IA1 & Right PcoA & Yes & Saccular & $13 \times 9$ \\
\hline IA 2 & BA & No & Saccular & $4 \times 3$ \\
\hline \multicolumn{5}{|l|}{ Test 9} \\
\hline IA1 & Righ MCA & Yes & Saccular & $9 \times 5$ \\
\hline IA2 & Right PcoA & No & Saccular & $6 \times 4$ \\
\hline \multicolumn{5}{|l|}{ Test 10} \\
\hline IA1 & AcoA & Yes & Saccular & $9 \times 3$ \\
\hline IA 2 & Left PcoA & No & Saccular & $4 \times 3$ \\
\hline
\end{tabular}

IA, intracranial aneurysm; SBP, systolic blood pressure; DBP, diastolic blood pressure; TG, triglyceride; LDL, low-density lipoprotein; HDL, high-density lipoprotein; PcoA, posterior communicating artery aneurysm; BA, basal artery aneurysm; MCA, middle cerebral artery; AcoA, anterior communicating artery. 
Table III. Top 10 upregulated and downregulated circRNAs.

\begin{tabular}{|c|c|c|c|c|c|c|}
\hline CircBase_ID & Direction of change & Host gene & Chromosomal location & Length (nt) & $\log 2 \mathrm{FC}$ & P-value \\
\hline hsa_circ_0135895 & Upregulation & PTK2 & Chr8 & 853 & 11.34 & 0.006 \\
\hline hsa_circ_0008911 & Upregulation & ZNF512 & Chr2 & 195 & 11.01 & 0.008 \\
\hline hsa_circ_0008122 & Upregulation & TCONS_12_00012420 & Chr19 & 223 & 10.90 & 0.008 \\
\hline hsa_circ_0074837 & Upregulation & PWWP2A & Chr5 & 965 & 10.75 & 0.009 \\
\hline hsa_circ_0078380 & Upregulation & SCAF8 & Chr6 & 1,159 & 10.64 & 0.010 \\
\hline hsa_circ_0093067 & Upregulation & CAMK1D & Chr10 & 192 & 10.53 & 0.010 \\
\hline hsa_circ_0037572 & Upregulation & SRRM2 & Chr16 & 93 & 10.33 & 0.012 \\
\hline hsa_circ_0089775 & Upregulation & PPP2R3B & Chrx & 1,253 & 10.28 & 0.012 \\
\hline hsa_circ_0000192 & Upregulation & GALNT2 & Chr1 & 248 & 9.40 & 0.022 \\
\hline hsa_circ_0131628 & Upregulation & SLC22A23 & Chr6 & 414 & 9.40 & 0.007 \\
\hline hsa_circ_0009076 & Downregulation & NRD1 & Chr1 & 228 & -10.33 & 0.021 \\
\hline hsa_circ_0000982 & Downregulation & LOC375190 & Chr2 & 395 & -10.16 & 0.024 \\
\hline hsa_circ_0001492 & Downregulation & ERBB2IP & Chr5 & 364 & -10.09 & 0.025 \\
\hline hsa_circ_0000698 & Downregulation & PHKB & Chr16 & 518 & -9.90 & 0.028 \\
\hline hsa_circ_0141172 & Downregulation & DAAM1 & Chr14 & 310 & -9.89 & 0.029 \\
\hline hsa_circ_0002665 & Downregulation & GDI2 & Chr10 & 343 & -9.86 & 0.029 \\
\hline hsa_circ_0001413 & Downregulation & FIP1L1 & Chr4 & 362 & -9.86 & 0.029 \\
\hline hsa_circ_0006208 & Downregulation & NPAT & Chr11 & 226 & -9.83 & 0.030 \\
\hline hsa_circ_0001936 & Downregulation & BRWD3 & Chrx & 676 & -9.82 & 0.030 \\
\hline hsa_circ_0126525 & Downregulation & SLAIN2 & Chr4 & 971 & -9.81 & 0.030 \\
\hline
\end{tabular}

chr, chromosome; hsa_circ, Homo sapiens circular RNA; FC, fold change.
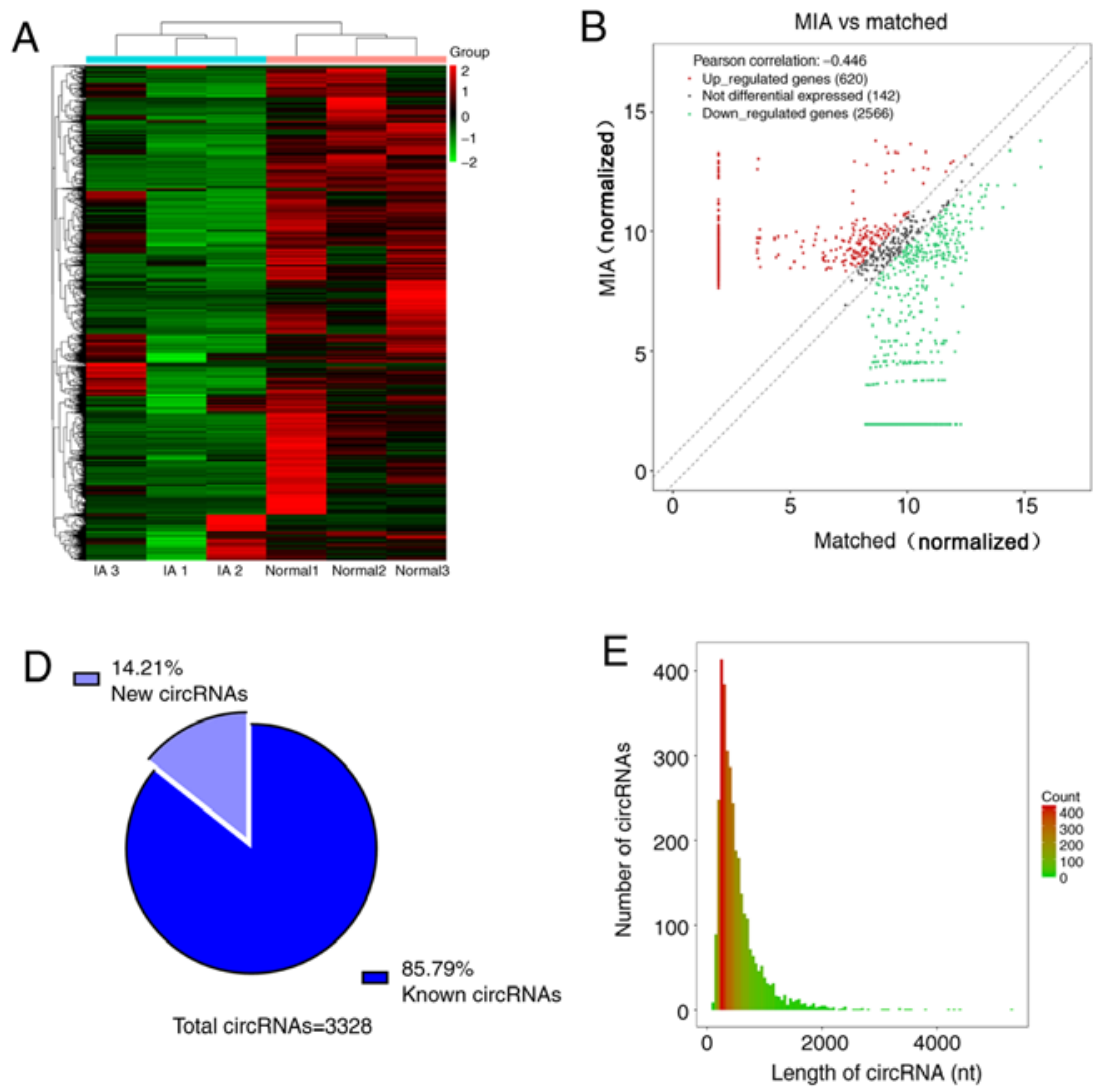

Figure 1. circRNA expression profiles of patients with MIA and matched individuals undergoing health examinations. (A) Hierarchical clustering, (B) scatter plot and $(C)$ volcano plot for the differentially expressed circRNAs between patients with MIA and matched individuals undergoing health examinations. (D) Proportion of previously known and newly discovered circRNAs detected by RNA-sequencing. (E) Length distribution of differentially expressed circRNAs. (F) Chromosomal location of circRNAs. circRNA, circular RNA; MIA, multiple intracranial aneurysms; chr, chromosome. 
Table IV. Predicted miRNAs for the selected significantly differential circRNAs in multiple intracranial aneurysm.

CircRNA ID

hsa_circ_0135895

hsa_circ_0000682

hsa_circ_0000690
miRNA

hsa-miR-619-3p, hsa-miR-4324, hsa-miR-5687, hsa-miR-3529-5p, hsa-miR-379-5p

hsa-miR-448, hsa-miR-1248, hsa-miR-302a-5p, hsa-miR-627-3p, hsa-miR-1248

hsa-miR-4726-3p, hsa-miR-4520-3p, hsa-miR-4514, hsa-miR-4692, hsa-miR-6842-3p

miRNA, microRNA; hsa_circ, Homo sapiens circular RNA.
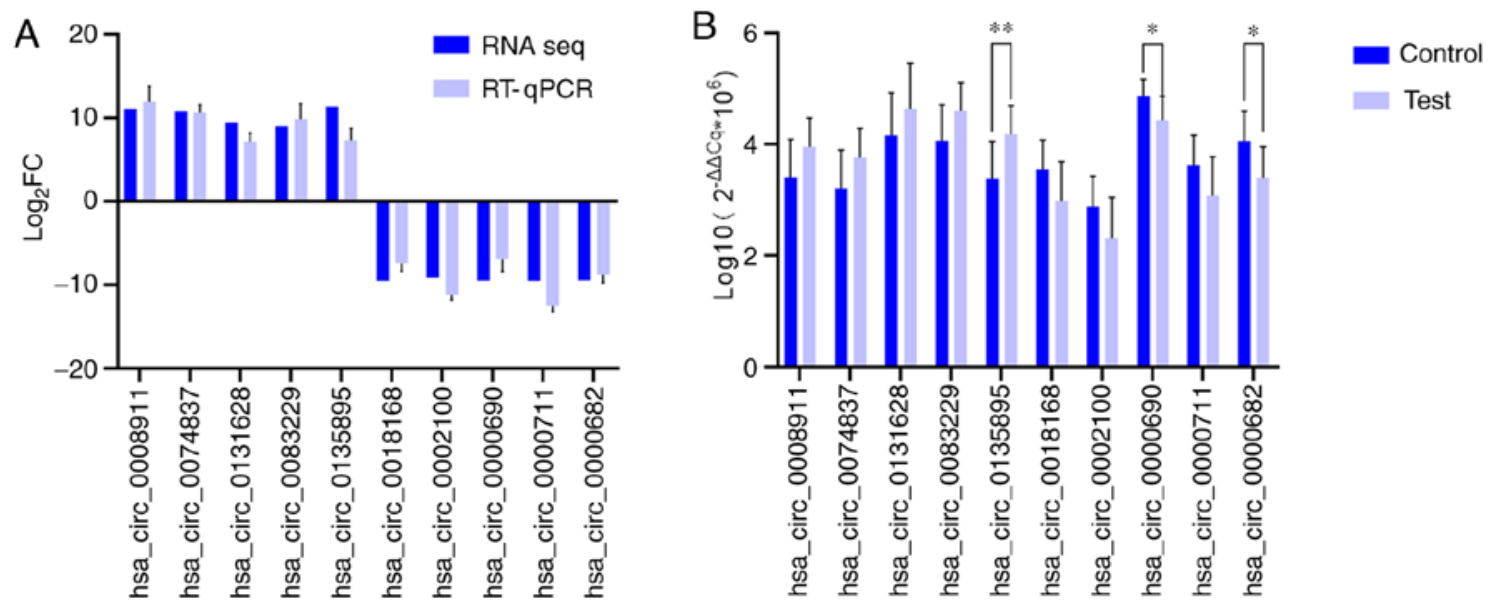

Figure 2. RT-qPCR validation. (A) Relative fold changes of circRNAs detected by RNA-seq and RT-qPCR. (B) RT-qPCR results for the 10 selected circRNAs in the test and control groups of the validation cohort. ${ }^{*} \mathrm{P}<0.05,{ }^{* *} \mathrm{P}<0.01$. circRNA, circular RNA; RT-qPCR, reverse transcription-quantitative PCR; FC, fold change; hsa_circ, Homo sapiens circular RNA; RNA-seq, RNA-sequencing.

hsa_circ_0135895] and 5 downregulated (hsa_circ_0018168, hsa_circ_0002100, hsa_circ_0000690, hsa_circ_0000711 and hsa_circ_0000682) circRNAs, were selected to confirm the sequencing data using RT-qPCR. As presented in Fig. 2A, the changes observed in all selected circRNAs were similar between circRNA sequencing and the RT-qPCR assays, which confirmed the accuracy of the sequencing. As indicated in Fig. 2B, hsa_circ_0135895, hsa_circ_0000690 and hsa_circ_0000682 were significantly differentially expressed between samples from 10 patients with MIA and 10 healthy individuals $(\mathrm{P}<0.05)$.

$G O$ and KEGG pathway analyses. GO enrichment and KEGG pathway analysis were performed with genes of a total of 3,328 differentially expressed circRNAs (Fig. 3). The top 10 most enriched GO terms by the upregulated and downregulated genes of identified circRNAs in the categories biological process (BP), cellular component and molecular function (MF) are presented in Fig. 3A and B. The top $10 \mathrm{GO}$ terms for the downregulated genes of identified circRNAs in the category BP were mainly involved in metabolic processes, while those in the category MF mainly involved different types of binding functions. The KEGG pathway analysis revealed that the upregulated genes of identified circRNAs mapped to 8 pathways, including 'thiamine metabolism', 'pyrimidine metabolism' and 'DNA replication' (Fig. 3C and E). Furthermore, the downregulated genes of identified circRNAs were mapped to 144 pathways, including 'leukocyte transendothelial migration' in Fig. 4A, 'natural killer cell mediated cytotoxicity' in Fig. 4B, 'T cell receptor signaling pathway' and 'NF- $\mathrm{KB}$ signaling pathway' (Fig. 3D and F). The 60 significantly differentially expressed circRNAs were also analyzed through GO enrichment and KEGG pathway analysis. The GO enrichment analysis was unsuccessful due to the low number of circRNAs. Only five pathways were obtained in the KEGG pathway analysis of the 60 host genes of the circRNAs identified, namely 'leukocyte transendothelial migration', 'natural killer cell mediated cytotoxicity', 'VEGF signaling pathway', 'axon guidance' and 'Wnt signaling pathway'.

miRNA prediction and ceRNA network construction. TargetScan, circBank and miRanda were utilized to predict miRNAs targeted by the differentially expressed circRNAs. The top 5 miRNAs predicted to be targeted by each of the three selected significantly differentially expressed circRNAs, which were all contained in the three databases, are presented in Table IV. The miRNA-mRNA regulatory relationships were further identified using miRTarBase and TargetScan. The miRNAs targeted by the circRNAs associated with 'leukocyte transendothelial migration' and their regulated mRNAs were further selected for ceRNA network construction. In this network, an upregulated circRNA (hsa_circ_0135895) and two downregulated circRNAs (hsa_circ_0000682 and hsa_circ_0000690) regulate protein tyrosine kinase 2 (PTK2), protein kinase C $\beta$ (PRKCB) and integrin subunit $\alpha \mathrm{L}$ (ITGAL), respectively, by sponging a 
A

Sig GO terms of DE genes
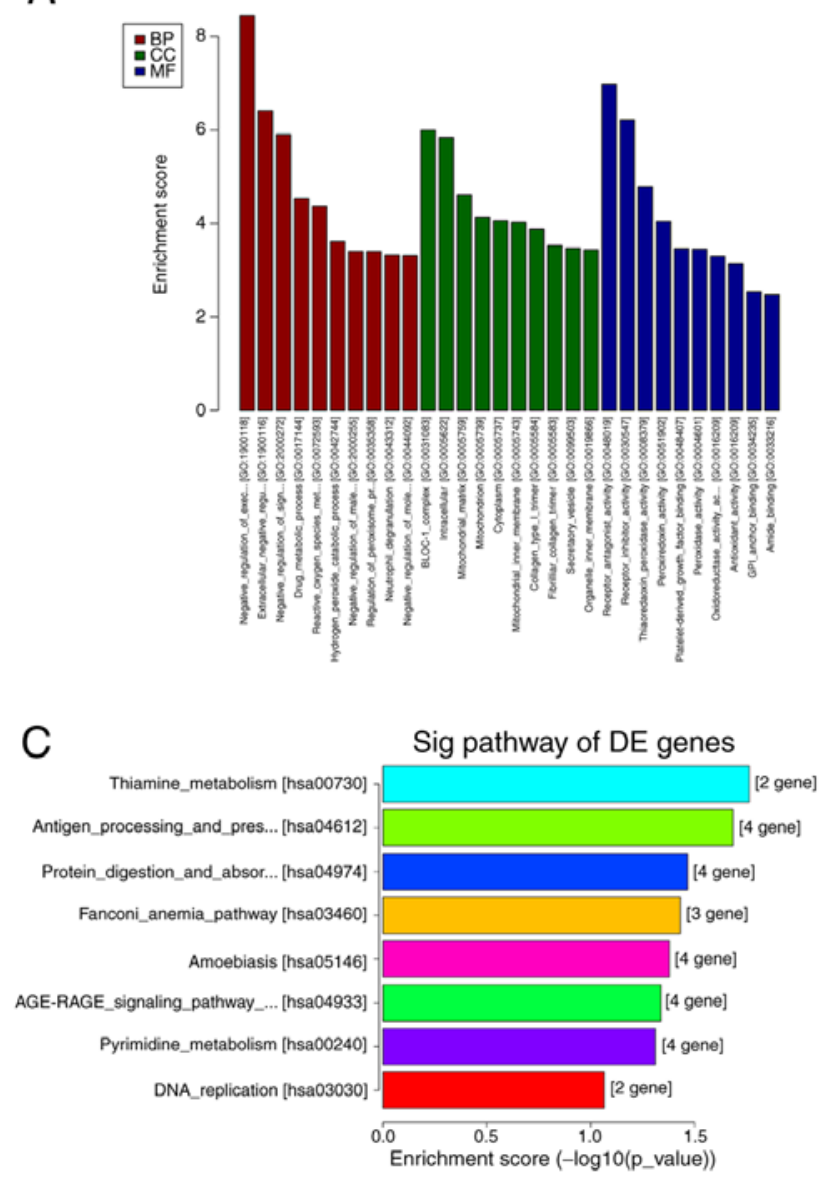

E

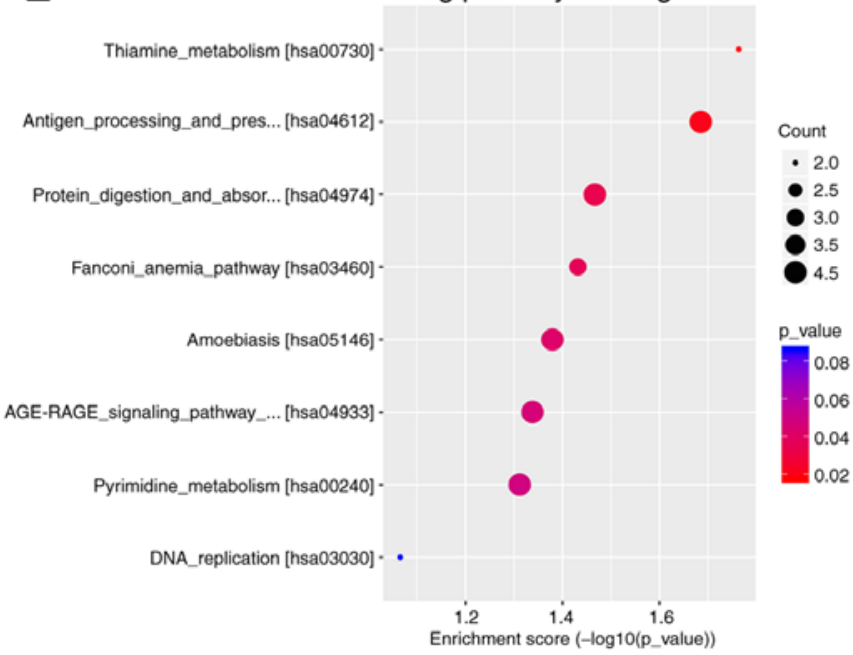

B

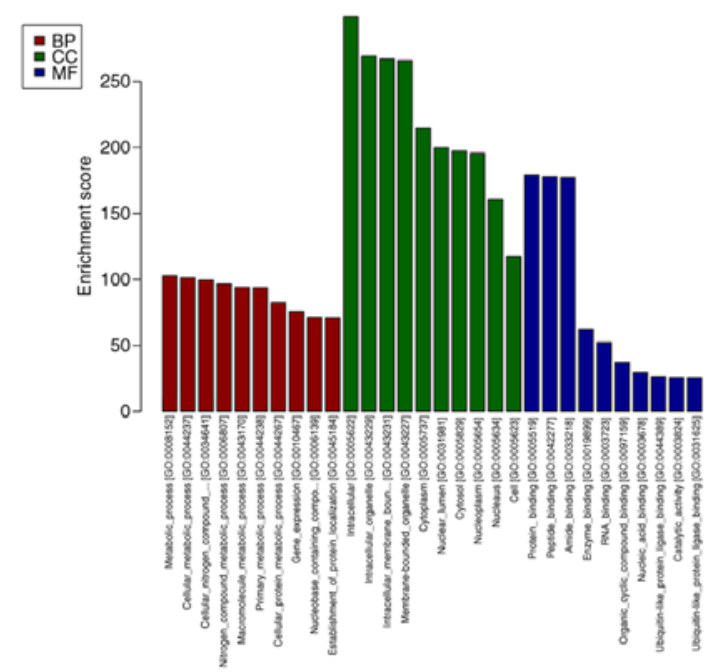

F

Leukocyte transendothelial migration [hsa04670]

Viral_carcinogenesis [hsa05203] .

Protein_processing_in_endop... [hsa04141] Natural killer cell mediated cytotoxicity [hsa04650]
Sig pathway of DE genes

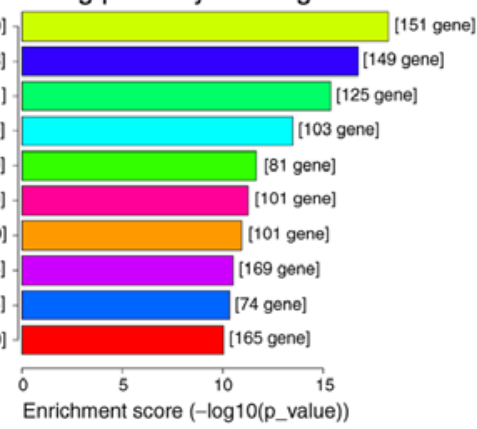

Sig pathway of DE genes

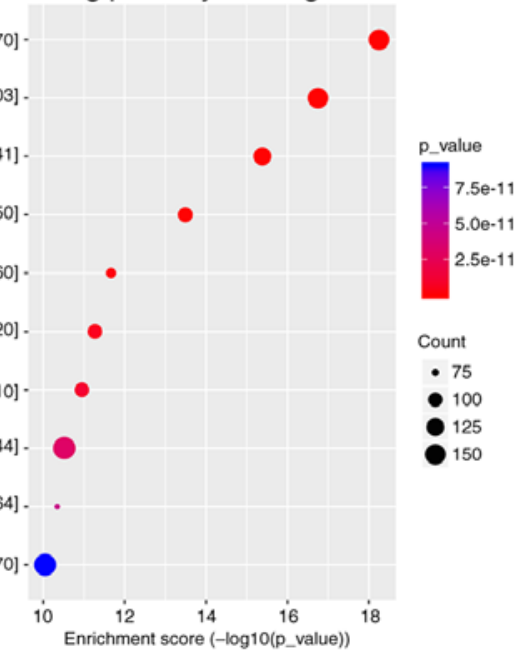

Figure 3. GO and KEGG pathway analysis. (A) Upregulated genes of identified circRNAs GO terms. (C and E) Pathways associated with the upregulated genes of identified circRNAs. (B) Downregulated genes of identified circRNAs GO terms. (D and F) Pathways associated with the downregulated genes of identified circRNAs. The significance of GO term enrichment and the pathways involved in the conditions was determined according to the P-value. A lower P-value (recommended cut-off, 0.05) suggests a higher significance of the GO terms and the pathways. GO, Gene Ontology; KEGG, Kyoto Encyclopedia of Genes and Genomes; Sig, significant; DE, differentially expressed; BP, biological process; CC, cellular component; MF, molecular function; hsa, Homo sapiens.

number of different miRNAs (Fig. 5). hsa-miR-4778-3p may combine with both hsa_circ_0135895 and hsa_circ_0000690, while hsa-miR-543 may combine with both hsa_circ_0135895 and hsa_circ_0000682. Several genes may combine with $>20$ predicted miRNAs, including ITGAL, IL2 inducible T-cell kinase, phosphatidylinositol-4,5-bisphosphate 3-kinase catalytic subunit $\alpha$, phospholipase $\mathrm{C} \gamma 1$, PRKCB,PTK2, protein tyrosine phosphatase non-receptor type 11, RAP1A member of RAS oncogene family, ras homolog family member $\mathrm{H}$, rho associated coiled-coil containing protein kinase 1 and signal-induced proliferation-associated 1. Among the three circRNAs, hsa_circ_0135895 sponged the most mRNAs. 

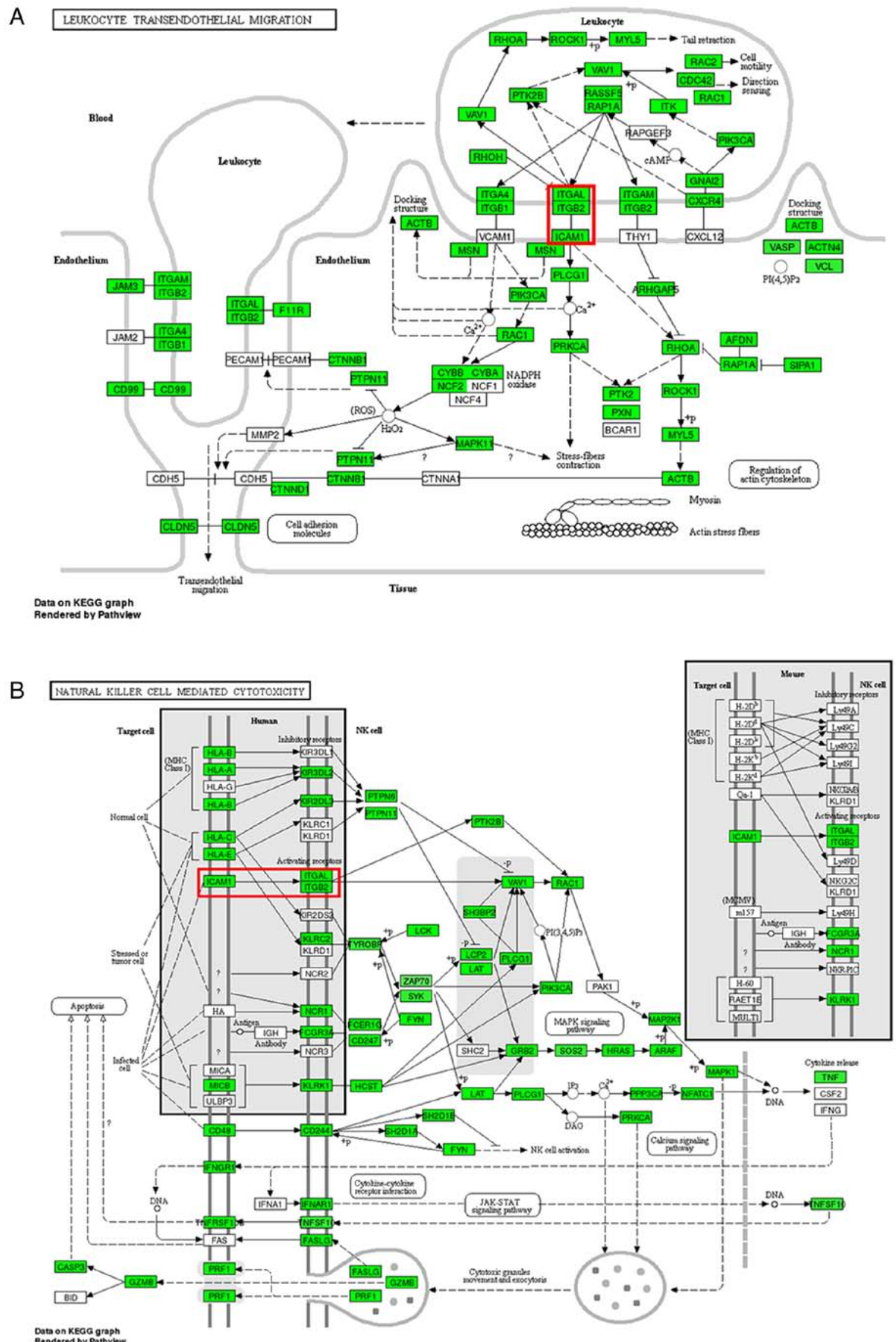

Figure 4. Pathways associated with the formation of multiple intracranial aneurysms. (A) Leukocyte transendothelial migration pathway. (B) Natural killer cell mediated cytotoxicity pathway. Green nodes represent gene enrichment of the pathway, while white nodes have no significance. The red square represents adhesion between intercellular cell adhesion molecule- 1 and the complex of integrin subunit $\alpha$ L/integrin subunit $\beta 2$. KEGG, Kyoto Encyclopedia of Genes and Genomes; NK, natural killer; ROS, reactive oxygen species; $\mathrm{H}_{2} \mathrm{O}_{2}$, hydrogen peroxide; DNA, deoxyribonucleic acid; cAMP, cyclic adenosine monophosphate; NADPH, nicotinamide adenine dinucleotide phosphate; DAG, diacylglycerol; IP3, inositol triphosphate. 


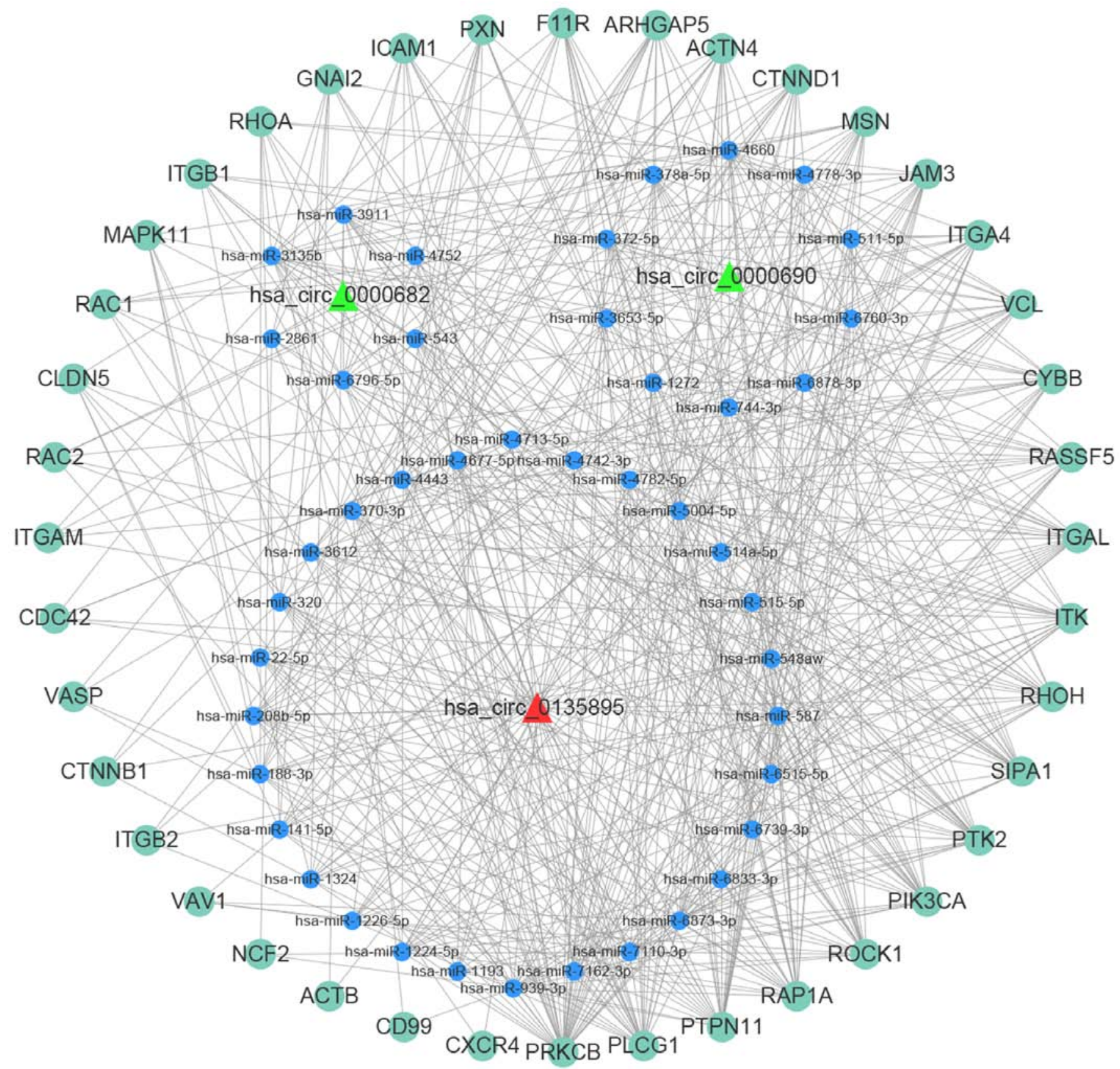

Figure 5. circRNA-miRNA-mRNA regulation network. The red triangle represents an upregulated circRNA (hsa_circ_0135895), the green triangles represent downregulated circRNAs (hsa_circ_0000682 and hsa_circ_0000690), the blue nodes represent miRNAs and the green nodes represent mRNAs. Hsa_circRNA, Homo sapiens circular RNA; miRNA, microRNA.

\section{Discussion}

MIA is an unpredictably and lethal disease. To the best of our knowledge, the specific molecular biological mechanisms of MIA have remained to be elucidated. IA, particularly MIA and familial aneurysms, may be hereditary (19). Previous studies have focused on genes associated with vascular smooth muscle cell dysfunction, extracellular matrix components and vascular inflammation; however, these genes may be associated with a change in risk of IA by $<5 \%(20,21)$. Gene expression is regulated by various factors, which are relatively reversible. As non-coding RNAs, a novel class of IncRNAs, circRNAs, are widely involved in gene transcription, post-transcriptional regulation and other biological processes. They have been identified and determined to be involved in neurologic diseases (22), particularly cerebrovascular disease (23).

In the present study, RNA-seq technology was used to profile differentially expressed circRNAs in peripheral blood mononuclear cells from patients with MIA. Samples from three pairs of patients with MIA and matched healthy individuals were selected to perform circRNA profiling. A total of 3,328 differentially expressed circRNAs were identified and 60 of these circRNAs were significantly differentially expressed, including 20 upregulated and 40 downregulated circRNAs. The reliability of the results was validated using RT-qPCR. The trends of all 10 selected circRNAs were the 
same (Fig. 2A), but some of them were not statistically significant (Fig. 2B). These results suggested that expression changes of these circRNAs are associated with the pathogenesis of MIA.

The GO enrichment and KEGG pathway analyses of the present study revealed the functional roles of the target genes. Since few pathways were identified for the upregulated genes, the present study focused on the pathways associated with the downregulated genes. It was revealed that most genes that were downregulated in MIA were mainly involved in inflammation, which is known to be critical for the pathogenesis of IA. This was similar to the results of Hao et al (24) and Huang et al (25). Inflammation of the arterial wall is one of the recognized mechanisms of IA (26). In addition, the aggregation of white blood cells and their adherence to inflammatory tissues are the basis of an inflammatory response (27). Inflammatory response and leukocyte infiltration are the pathological basis for the development of IA $(28,29)$. Infiltration of inflammatory cells is the primary pathological change during the early stages of IA (30). circRNAs have been demonstrated to serve important roles in inflammation, including the regulation of inflammation-associated genes, recruitment of macrophages and modulation of crucial signaling pathways (31). The present results suggested that several inflammation-related biological mechanisms and pathways in MIA are potentially regulated by circRNAs. The most affected pathway was the 'leukocyte transendothelial migration' in Fig. 4A. Due to the blood-brain barrier, there is almost no peripheral blood lymphocyte aggregation in normal brain tissues. However, the adhesion between lymphocyte function-associated antigen-1 (LFA-1)/intercellular cell adhesion molecule-1 (ICAM-1) in the pathway 'leukocyte transendothelial migration' serves an important role in the migration and infiltration of lymphocytes into cerebrovascular wall tissues (32). 'Natural killer cell mediated cytotoxicity' in Fig. 4B was the other signaling pathway associated with the downregulated genes and was associated with MIA formation and development. In addition, studies have demonstrated that natural killer cells intensively infiltrate in abdominal aortic aneurysm and damage the vessel by inducing cytotoxicity in arterial smooth muscle cells (33).

Adhesion between LFA-1 and ICAM-1 is involved in both 'leukocyte transendothelial migration' and 'Natural killer cell mediated cytotoxicity' pathways in Fig. 4. LFA-1 is expressed on the surfaces of lymphocytes, neutrophils, monocytes and macrophages. As a member of the integrin $\beta 2$ family, LFA- 1 is composed of an $\alpha$ and a $\beta$ subunit. The $\alpha$ subunit, also known as CD11a antigen, is encoded by ITGAL, and the $\beta$ subunit, also known as CD18, is encoded by integrin subunit $\beta 2$ (34). CD11a, which may be transcriptionally regulated by hsa circ_0000690, is the binding site for ICAM-1, enabling stable immune adhesion between LFA-1 and ICAM-1.

'Leukocyte transendothelial migration' may be a key biological event in MIA. In the present study, the KEGG pathway 'leukocyte transendothelial migration' was enriched by 151 genes targeted by differentially expressed circRNAs. Most circRNAs have been identified to interact with miRNAs via fewer binding sites, acting as miRNA sponges to regulate transcription. Therefore, the ceRNA mechanism of certain circRNAs associated with 'leukocyte transendothelial migration' was explored. For this, three circRNAs that interact with certain miRNAs that transcriptionally regulate migration were selected (hsa_circ_0135895, hsa_circ_0000682 and hsa_circ_0000690) and annotated, since the validation experiment confirmed that these three circRNAs were significantly different between the cases and controls. Furthermore, a ceRNA network was constructed based on the selected circRNAs. Subsequently, circRNA-miRNA interactions were assessed to further reveal the roles of circRNAs as miRNA sponges.

It is worth mentioning that the present results demonstrated that the downregulated genes were over-represented in pathways associated with inflammation. It is known that inflammation is an important factor in the occurrence and development of IAs. Therefore, the opposite results would be expected. The observed negative association appeared to be the opposite of the expected trend. The exact mechanisms responsible for these results remain elusive and it was not possible to offer a reasonable explanation based on the results of the present study. The most recent studies have focused on upregulated circRNAs due to the ceRNA mechanism (35-37). circRNAs are widely involved in gene transcription, post-transcriptional regulation and other biological processes with complex and unknown mechanisms. The ceRNA mechanism is just one classical mechanism. Only a small number of studies have investigated downregulated genes; however, increasing attention is being paid to these $(38,39)$. Therefore, the relationships among downregulated genes, inflammation and MIA, as well as the mechanisms underlying their associations, deserve further investigation.

In the present study, circRNA expression profiles were linked to MIA development to predict a regulatory role of circRNAs in MIA formation, thereby expanding the knowledge of MIA. However, the present study had certain limitations. First, only circRNAs associated with 'leukocyte transendothelial migration' were used to construct the ceRNA network, while circRNAs associated with other pathways may also have an effect on transcriptional regulation in MIA. Therefore, further investigation including more circRNAs is required. In addition, the effects of circRNAs on miRNAs and mRNAs were determined by computational analyses and remain to be experimentally demonstrated. Further biological in vitro and in vivo experiments are required, including validation of the expression of target miRNAs. As another limitation, both ruptured and unruptured IAs were included in the research objective. Differences in circRNA expression in MIA may be caused by not only IA itself but also subarachnoid hemorrhage. Whether the results may apply to unruptured MIA or single IA with ruptured status warrants further investigation. Finally, only samples from female patients were used in the present study. There may be certain significant differentially expressed circRNAs in the Y chromosome that the present study was not able to assess.

To elucidate how circRNAs transcriptionally regulate MIA formation and to identify biomarkers of MIA, research in this field based on the present results may be performed.

In conclusion, to the best of our knowledge, the present study was the first to identify differential expression profiles of circRNAs in MIA. Most downregulated genes were mainly involved in inflammation. The KEGG pathway 'leukocyte transendothelial migration' may be critical for the pathogenesis of MIA. circRNAs involved in this pathway, particularly 
hsa_circ_0135895, hsa_circ_0000682 and hsa_circ_0000690, should be further explored in the future.

\section{Acknowledgements}

The authors would like to thank Dr Yingying Lin (Department of Neurosurgery, Ren Ji Hospital Affiliated to the School of Medicine of Shanghai Jiao Tong University, Shanghai, China), who supported the present study in terms of experimental design and technology regarding RNA isolation and purification.

\section{Funding}

The present study was supported by the Basic Research and Young Talent Programs Foundation of the Science and Technology Department of Longyan City (grant no. 2018LYF8019).

\section{Availability of data and materials}

The datasets used or analyzed during the current study are available from the corresponding author on reasonable request. They are available from the Gene Expression Omnibus (GEO) repository (series entry, GSE159631; (https://www.ncbi.nlm. nih.gov/geo/query/acc.cgi?acc=GSE159631).

\section{Authors' contributions}

$\mathrm{HC}$ and $\mathrm{YH}$ participated in the design of the present study and performed the statistical analysis. HC, JC and XL performed the experiments and data acquisition. HC, JC and YH confirmed the authenticity of the raw data. YH, TL, PQ and SQ analyzed and interpreted the data. YH drafted the manuscript and obtained funding. HC, JC, TL, PQ and SQ revised the manuscript. All authors read and approved the final manuscript.

\section{Ethics approval and consent to participate}

This study was approved by the Scientific Research Ethics Committee of Longyan First Hospital Affiliated to Fujian Medical University (Longyan, China; Ethical Review Scientific Research no. 003; 2018). Written informed consent was obtained from the patients or their relatives for participation in this study, including the RNA-seq cohort and validation cohort.

\section{Patient consent for publication}

Not applicable.

\section{Competing interests}

The authors declare that they have no competing interests.

\section{References}

1. Nieuwkamp DJ, Setz LE, Algra A, Linn FH, de Rooij NK and Rinkel GJ: Changes in case fatality of aneurysmal subarachnoid haemorrhage over time, according to age, sex, and region: A meta-analysis. Lancet Neurol 8: 635-642, 2009.
2. Brown RD Jr, Huston J, Hornung R, Foroud T, Kallmes DF, Kleindorfer D, Meissner I, Woo D, Sauerbeck L and Broderick J: Screening for brain aneurysm in the Familial Intracranial Aneurysm study: Frequency and predictors of lesion detection. J Neurosurg 108: 1132-1138, 2008.

3. Bo L, Wei B, Wang Z, Kong D, Gao Z and Miao Z: Screening of Critical Genes and MicroRNAs in Blood Samples of Patients with Ruptured Intracranial Aneurysms by Bioinformatic Analysis of Gene Expression Data. Med Sci Monit 23: 4518-4525, 2017.

4. Wei L, Wang Q, Zhang Y, Yang C, Guan H, Chen Y and Sun Z: Identification of key genes, transcription factors and microRNAs involved in intracranial aneurysm. Mol Med Rep 17: 891-897, 2018.

5. Korostynski M, Morga R, Piechota M, Hoinkis D, Golda S, Dziedzic T, Slowik A, Moskala M and Pera J: Inflammatory Responses Induced by the Rupture of Intracranial Aneurysms Are Modulated by miRNAs. Mol Neurobiol 57: 988-996, 2020.

6. Li H, Wang W, Zhang L, Lan Q, Wang J, Cao Y and Zhao J: Identification of a Long Noncoding RNA-Associated Competing Endogenous RNA Network in Intracranial Aneurysm. World Neurosurg 97: 684-692.e4, 2017.

7. Wu C, Song H, Wang Y, Gao L, Cai Y, Cheng Q, Chen Y, Zheng Z, Liao Y, Lin J, et al: Long non-coding RNA TCONS_00000200 as a non-invasive biomarker in patients with intracranial aneurysm. Biosci Rep 39: 39, 2019.

8. Kulcheski FR, Christoff AP and Margis R: Circular RNAs are miRNA sponges and can be used as a new class of biomarker. J Biotechnol 238: 42-51, 2016.

9. 't Hoen PA, Ariyurek Y, Thygesen HH, Vreugdenhil E, Vossen RH, de Menezes RX, Boer JM, van Ommen GJ and den Dunnen JT: Deep sequencing-based expression analysis shows major advances in robustness, resolution and inter-lab portability over five microarray platforms. Nucleic Acids Res 36: e141, 2008.

10. Khan S and Kaihara KA: Single-Cell RNA-Sequencing of Peripheral Blood Mononuclear Cells with ddSEQ. Methods Mol Biol 1979: 155-176, 2019.

11. Jeck WR and Sharpless NE: Detecting and characterizing circular RNAs. Nat Biotechnol 32: 453-461, 2014.

12. Li HM, Dai YW, Yu JY, Duan P, Ma XL, Dong WW, Li N and Li HG: Comprehensive circRNA/miRNA/mRNA analysis reveals circRNAs protect against toxicity induced by BPA in GC-2 cells. Epigenomics 11: 935-949, 2019.

13. Wang Y, Wang Y, Li Y, Wang B, Miao Z, Liu X and Ma Y: Decreased expression of circ_0020397 in intracranial aneurysms may be contributing to decreased vascular smooth muscle cell proliferation via increased expression of miR-138 and subsequent decreased KDR expression. Cell Adhes Migr 13: 220-228, 2019.

14. Livak KJ and Schmittgen TD: Analysis of relative gene expression data using real-time quantitative PCR and the 2(-Delta Delta C(T)) Method. Methods 25: 402-408, 2001.

15. Agarwal V, Bell GW, Nam JW and Bartel DP: Predicting effective microRNA target sites in mammalian mRNAs. eLife 4: 4, 2015.

16. Glažar P, Papavasileiou P and Rajewsky N: circBase: A database for circular RNAs. RNA 20: 1666-1670, 2014.

17. Chou CH, Shrestha S, Yang CD, Chang NW, Lin YL, Liao KW, Huang WC, Sun TH, Tu SJ, Lee WH, et al: miRTarBase update 2018: A resource for experimentally validated microRNA-target interactions. Nucleic Acids Res 46D: D296-D302, 2018.

18. Maass PG, Glažar P, Memczak S, Dittmar G, Hollfinger I, Schreyer L, Sauer AV, Toka O, Aiuti A, Luft FC, et al: A map of human circular RNAs in clinically relevant tissues. J Mol Med (Berl) 95: 1179-1189, 2017.

19. Caranci F, Briganti F, Cirillo L, Leonardi M and Muto M: Epidemiology and genetics of intracranial aneurysms. Eur J Radiol 82: 1598-1605, 2013.

20. Alg VS, Sofat R, Houlden H and Werring DJ: Genetic risk factors for intracranial aneurysms: A meta-analysis in more than 116,000 individuals. Neurology 80: 2154-2165, 2013.

21. Hussain I, Duffis EJ, Gandhi CD and Prestigiacomo CJ: Genome-wide association studies of intracranial aneurysms: An update. Stroke 44: 2670-2675, 2013.

22. Shao Y and Chen Y: Roles of Circular RNAs in Neurologic Disease. Front Mol Neurosci 9: 25, 2016.

23. van Rossum D, Verheijen BM and Pasterkamp RJ: Circular RNAs: Novel Regulators of Neuronal Development. Front Mol Neurosci 9: 74, 2016.

24. Hao Z, Li Y, Yu N, Zhao Y, Hu S, Liu Z and Li M: Analysis of differentially expressed circular RNAs in endothelial cells under impinging flow. Mol Cell Probes 51: 101539, 2020. 
25. Huang Q, Huang QY, Sun Y and Wu S: High-Throughput Data Reveals Novel Circular RNAs via Competitive Endogenous RNA Networks Associated with Human Intracranial Aneurysms. Med Sci Monit 25: 4819-4830, 2019.

26. Signorelli F, Sela S, Gesualdo L, Chevrel S, Tollet F, Pailler-Mattei C, Tacconi L, Turjman F, Vacca A and Schul DB: Hemodynamic Stress, Inflammation, and Intracranial Aneurysm Development and Rupture: A Systematic Review. World Neurosurg 115: 234-244, 2018

27. Chidlow JH Jr, Glawe JD, Alexander JS and Kevil CG: VEGF164 differentially regulates neutrophil and $\mathrm{T}$ cell adhesion through ItgaL- and ItgaM-dependent mechanisms. Am J Physiol Gastrointest Liver Physiol 299: G1361-G1367, 2010.

28. Hoh BL, Rojas K, Lin L, Fazal HZ, Hourani S, Nowicki KW, Schneider MB and Hosaka K: Estrogen Deficiency Promotes Cerebral Aneurysm Rupture by Upregulation of Th17 Cells and Interleukin-17A Which Downregulates E-Cadherin. J Am Heart Assoc 7: 7, 2018.

29. Sawyer DM, Pace LA, Pascale CL, Kutchin AC, O'Neill BE, Starke RM and Dumont AS: Lymphocytes influence intracranial aneurysm formation and rupture: Role of extracellular matrix remodeling and phenotypic modulation of vascular smooth muscle cells. J Neuroinflammation 13: 185, 2016.

30. Liu Y, Zhang Y, Dai D and Xu Z: Expression of NF-kappaB, MCP-1 and MMP-9 in a Cerebral Aneurysm Rabbit Model. Can J Neurol Sci 41: 200-205, 2014.

31. Dou C, Cao Z, Yang B, Ding N, Hou T, Luo F, Kang F, Li J, Yang $\mathrm{X}$, Jiang $\mathrm{H}$, et al: Changing expression profiles of IncRNAs, mRNAs, circRNAs and miRNAs during osteoclastogenesis. Sci Rep 6: 21499, 2016.

32. Faveeuw C, Di Mauro ME, Price AA and Ager A: Roles of alpha(4) integrins/VCAM-1 and LFA-1/ICAM-1 in the binding and transendothelial migration of $\mathrm{T}$ lymphocytes and $\mathrm{T}$ lymphoblasts across high endothelial venules. Int Immunol 12: 241-251, 2000.
33. Hinterseher I, Schworer CM, Lillvis JH, Stahl E, Erdman R, Gatalica Z, Tromp G and Kuivaniemi H: Immunohistochemical analysis of the natural killer cell cytotoxicity pathway in human abdominal aortic aneurysms. Int J Mol Sci 16: 11196-11212, 2015.

34. Yang H, Graham LC, Reagan AM, Grabowska WA, Schott WH and Howell GR: Transcriptome profiling of brain myeloid cells revealed activation of Itgal, Trem1, and Spp1 in western diet-induced obesity. J Neuroinflammation 16: 169, 2019.

35. Zhu Z,Li Y,Liu W,He J,Zhang L,LiH,LiP and Lv L: Comprehensive circRNA expression profile and construction of circRNA-associated ceRNA network in fur skin. Exp Dermatol 27: 251-257, 2018.

36. Wu J, Liu S, Xiang Y, Qu X, Xie Y and Zhang X: Bioinformatic Analysis of Circular RNA-Associated ceRNA Network Associated with Hepatocellular Carcinoma. BioMed Res Int 2019: 8308694, 2019.

37. Cao M, Zhang L, Wang JH, Zeng H, Peng Y, Zou J, Shi J, Zhang L, Li Y, Yoshida S, et al: Identifying circRNA-associated-ceRNA networks in retinal neovascularization in mice. Int J Med Sci 16: 1356-1365, 2019.

38. Li X, Ding J, Wang X, Cheng Z and Zhu Q: NUDT21 regulates circRNA cyclization and ceRNA crosstalk in hepatocellular carcinoma. Oncogene 39: 891-904, 2020.

39. Li C, Li M and Xue Y: Downregulation of CircRNA CDR1as specifically triggered low-dose Diosbulbin-B induced gastric cancer cell death by regulating miR-7-5p/REG $\gamma$ axis. Biomed Pharmacother 120: 109462, 2019.

This work is licensed under a Creative Commons Attribution-NonCommercial-NoDerivatives 4.0 International (CC BY-NC-ND 4.0) License. 